\title{
Aggressive Blood Pressure Control in Intracerebral Hemorrhage (the Abc-Ich Study)—A Pilot Study
}

\author{
Harold Andrew Sloas ${ }^{1}$, Raja Malkani², Navdeep Shangha' ${ }^{1}$, Yashwant Chathampally ${ }^{1}$ \\ ${ }^{1}$ The Department of Emergency Medicine, The University of Texas Health Science Center at Houston, Houston, \\ USA \\ ${ }^{2}$ The University of Texas, Austin Texas, USA \\ Email: harold.sloas@uky.edu, navdeepsangha1@gmail.com, rajamalkani@gmail.com, \\ Yashwant.Chathampally@uth.tmc.edu
}

Received 25 January 2014; revised 22 February 2014; accepted 23 March 2014

Copyright (C) 2014 by authors and Scientific Research Publishing Inc.

This work is licensed under the Creative Commons Attribution International License (CC BY). http://creativecommons.org/licenses/by/4.0/

c) $\underset{\mathrm{EY}}{\mathrm{B}}$ Open Access

\section{Abstract}

Spontaneous intracerebral hemorrhage (ICH) accounts for one fifth of all strokes and is associated with an extremely high rate of morbidity and mortality. Affecting greater than 1 million people a year, ICH will leave the majority of its' patients significantly disabled or dead. An initially high systolic blood pressure upon presentation is associated with hematoma expansion, peri-hematoma expansion, and increased mortality. The relationship between blood pressure, the degree of blood pressure control and hematoma expansion has yet to be defined, but the literature has observed a relationship between tightly controlled blood pressures and decreased hematoma expansion. There have been many proposed mechanisms to explain this effect. Larger initial hematomas may lend greater hydrostatic forces and this could result in greater total hematoma volume, and greater surrounding edema. Recent literature has suggested that blood pressure reductions in acute ICH may be tolerated because of reduced metabolism, and preserved autoreguation in the peri-hematoma region. The volume of the hematoma is a critical determinant of mortality and functional outcome after intracerebral hemorrhage, and early hematoma growth is an important cause of neurologic deterioration. An increase in volume of more than thirty-three percent is detectable on repeated computed tomography (CT) in thirty-eight percent of patients initially scanned within the first three hours of onset of symptoms; in two thirds of these cases this change is noticeable on CT within the first hour. This supports the hypothesis that early aggressive blood pressure optimization would decrease hematoma size and edema. This is further supported by the fact that patients with high blood pressure and acute intracerebral hemorrhage have worse outcomes than their counterparts. We hypothesize that prompt and aggressive, early blood pressure reduction in emergency department patients with acute spontaneous intracerebral hemorrhage will result in a reduction of early hematoma growth. The study institution is a large urban emer- 
gency department and tertiary care stoke center, with over 55,000 emergency department visits per year. This prospective cohort study compared the results and outcomes observed within the enrolled prospective study population, to the results and outcomes of a matched historical cohort population (future patients with intracranial hemorrhage that did not receive the ABC-ICH protocol). Methods and Material: A nicardipine infusion was administered to optimize blood pressure in all patients presenting with intracerebral hemorrhage with a target mean arterial pressure (MAP) of 80 - 110. Hematoma volume (primary outcome measure) was measured on cat scans at time of presentation and at twenty-four hours. The hematoma volume in the enrolled prospective study population was compared to those of a matched cohort (patients with intracranial hemorrhage that did not receive the ABC-ICH protocol following the conclusion of the study). Results: One hundred total patients were enrolled into the study. Fifty patients were enrolled prospectively in the study and matched to a similar group of fifty cohort patients. The difference in the mean change of hematoma volume at twenty-four hours was $7.29 \mathrm{ml}$ (control) and $2.84 \mathrm{ml}$ (study). The result was an absolute decrease in hematoma size of $4.45 \mathrm{ml}$ in the group treated aggressively with nicardapine for blood pressure reduction within one hour of their initial presentation. Conclusions: These results support the previous research suggesting that aggressive blood pressure control in intracerebral hemorrhage reduces hematoma growth, however the clinical benefit of such a reduction will have to be evaluated in ongoing research.

\section{Keywords}

ICH; Intrecerebral Hemorrhage; Stroke; Intracranial Bleeding; Stroke Treatment; Intracerebral Hemorrhage Treatement; Nicardapine

\section{Introduction}

Spontaneous intracerebral hemorrhage (ICH) accounts for one fifth of all strokes and is associated with an extremely high rate of morbidity and mortality. ICH affects greater than 1 million people a year and the majority of patients suffer significant disability or death [1] [2]. An initially high systolic blood pressure upon presentation is associated with hematoma expansion [3] [4], peri-hematoma expansion [5], and increased mortality [6]. The relationship between blood pressure, the degree of blood pressure control and hematoma expansion has yet to be defined, but the literature has observed a relationship between tightly controlled blood pressures and decreased hematoma expansion [7]-[9]. There have been many proposed mechanisms to explain this effect. Larger initial hematomas may lend greater hydrostatic forces and this could result in greater total hematoma volume, and greater surrounding edema [10] [11].

Recent literature has suggested that blood pressure reductions in acute ICH may be tolerated because of reduced metabolism [12], and preserved autoreguation in the peri-hematoma region [13]. Currently, the American Stroke Association Stroke Council [14] and the European Stroke Initiative Guidelines [15] recommend maintaining SBP $<180 \mathrm{mmHg}$ in the acute phase of ICH. Both guidelines have allowed leeway for more aggressive approaches to blood pressure control in patients without clinical signs of increased intracranial pressure or chronic hypertension [14] [15].

The ABC ICH trial was designed to answer the question: can early aggressive blood pressure reduction limit the size of hematoma expansion in acute ICH? We hypothesized that by initiating a nicardipine infusion within the first hour of presentation to the ED we could rapidly achieve a MAP of 80 - 110 and thus decrease overall hematoma volumes. Those volumes were measured on the initial cat scan (CT) of the brain and a twenty-four hour follow-up brain CT.

\section{Methods and Materials}

This prospective cohort study compared the results and outcomes observed within the enrolled prospective study population, to the results and outcomes of a matched historical cohort population (future patients with intracranial hemorrhage that did not receive the ABC-ICH protocol). Historical data was collected from medical 
record reviews of patients with ICH as recorded in the stroke registry, as well as from previous acute ICH treatment trials.

Setting and Selection of patients:

The study institution is a large urban emergency department and tertiary care stoke center, with over 55,000 emergency department visits per year, and estimated average of 150 admissions per year for acute intracranial hemorrhage. We included all patients older than 18 years of age who presented to the emergency department within four hours of onset of signs and symptoms of an acute intracranial hemorrhage. To be eligible for inclusion in this study, patients had to satisfy all of the following criteria.

We enrolled patients with a primary diagnosis must have been acute stroke due to spontaneous ICH. This was defined as the sudden occurrence of bleeding into the parenchyma of the brain, which could extend into the ventricles and, in rare cases, into the subarachnoid space. The hemorrhage had to be confirmed by CT scan. No patients with ICH known or thought to be secondary to medical treatment (eg, antithrombotic therapy) were eligible.

The initial systolic blood pressure measurement had to be $\leq 220 \mathrm{mmHg}$ and $\geq 150 \mathrm{mmHg}$. Patients with initial systolic blood pressure levels outside this range [i.e. $<150$ or $>220 \mathrm{mmHg}$ ] were still randomized but were not eligible for enrolment unless they had two subsequent blood pressures inside the appropriate range. Moreover, patients with systolic SBP $>220 \mathrm{mmHg}$ were eligible to receive initial blood pressure lowering agent and then enrolled, provided systolic BP was $<220 \mathrm{mmHg}$ within three hours of symptom onset. If the precise timing of the first signs and symptoms of the qualifying event were unknown, then the time of onset was taken as the last time at which the patient was known to be well.

All patients had to be eligible for aggressive blood pressure treatment and qualify for admission to a monitored facility such as an acute stroke unit, high dependency unit or intensive care unit. It was recognized that many ICH patients were designated "Not For Resuscitation" or "Do Not Resuscitate" after appropriated consultation with family members. This in and of itself was not a contraindication to enrolment in the trial, as long as the management was otherwise consistent with the study protocol.

Patients were not eligible if they had any of the following: A known definite contraindication to an intensive BP lowering regimen (eg severe carotid, vertebral or cerebral arterial stenosis, known Moya Moya disease or Takayasu's arteritis, high-grade stenotic valvular heart disease, or severe renal failure); a known definite indication for an intensive BP lowering regimen as (or more) intensive than the active treatment arm of this study (eg, hypertensive encephalopathy, or aortic dissection); any definite evidence that the ICH was secondary to a structural abnormality in the brain (eg, an AVM, intracranial aneurysm, tumour, trauma, or previous cerebral infarction) or a previous thrombolysis; a previous ischemic stroke within 30 days; a very high likelihood that the patient would die within the ensuing twenty-four hours on the basis of clinical and/or radiological criteria (eg, massive hematoma with mid-line shift of hemisphere or deep coma on presentation, defined by Glasgow Coma Scale Score of three to five); a known advanced dementia or significant pre-stroke disability; a concomitant medical illness that would interfere with outcome assessments and follow-up (eg, advanced cancer or respiratory disease); the patient was already scheduled for surgical evacuation of hematoma, previous participation in this trial or current participation in another investigational drug trial; a high likelihood that the patient would not adhere to the study treatment and follow-up regimen; pregnant patients were also excluded.

All patients presenting to the emergency department and meeting the inclusion criteria were enrolled in this study. In the initial emergency department assessment stage, standard emergent medical requirements were assessed and addressed before enrollment. Intra-venous access, a 12 lead ECG, laboratory data, and bedside glucose testing were obtained. Per the hospital protocol the twenty-four hour in-house stroke team (to include a stroke fellow or attending, emergency or neuro-radiologist, and CT technician) were alerted upon arrival of the patient and present for the initial exam and testing.

The study design was not designed as a new or alternative therapeutic management strategy for acute intracranial hemorrhage. The methods section outlines the standard process for identifying and managing acute ICH from the ED. For that reason, we were given a waiver of informed consent by the local IRB.

In the immediate neurologic assessment phase a standard emergency room protocol was followed for review of the relevant patient history and performance of a physical exam (with an appropriate emphasis placed on the neurologic exam). The patient's level of consciousness was assessed using the Glasgow Coma Scale (GCS), with repeated GCS measurements for neurologic change on an hourly basis. Stroke severity was assessed using the National Institute of Health Stroke Scale (NIHSS) with repeat assessment of stroke severity/progression on 
an hourly basis using the NIHSS, a modified Rankin Score and ICH score.

A non-contrast CT scan of the brain was ordered, and preformed using a VCT 64-slice CT scanner. The goal was to have a non-contrast CT of the brain performed in less than 25 minutes from the patient's arrival and read by an emergency trained radiologist, neuro-radiologist or stroke neurologist within forty-five minutes of the patient's arrival in emergency department. Volumes of intracranial hemorrhage were calculated, from the initial brain $\mathrm{CT}$ using the $\mathrm{A} * \mathrm{~B} * \mathrm{C} / 2$ method, by a blinded radiologist or un-blinded neuro-radiologist.

After an acute intracranial hemorrhage was confirmed, intravenous nicardipine, with an allowable initial bolus dose of $0.01 \mathrm{mg} / \mathrm{kg}$ (with a dose no less than $0.5 \mathrm{mg}$ and no more than $1 \mathrm{mg}$ ) was administered in the emergency room. Labetolol was the alternative medication chosen by the admitting neurology service or pre-hospital personnel to obtain a blood pressure adequate for enrollment. A nicardipine infusion was then started to obtain blood pressure reduction. A time to initiation for the nicardipine infusion of less than sixty minutes from the patient's arrival time to the ED was required to continue enrollment. The blood pressure target was a mean arterial pressure (MAP) of 80 - 110. Blood pressures were monitored using an automated cuff every five minutes until the blood pressure had stabilized and then every ten minutes until the patient was transferred to the stroke unit or ICU. Regular physical exams were repeated to evaluate the patient's neurologic status. Those assessments, as previously outlined, were performed in the emergency department and continued while the patient was in the stroke unit. A final Modified Rankin Score was assigned to the patient at time of discharge or death (Table 1). Anticonvulsant medications were only used to treat active seizures. Coagulopathies were corrected depending on individual patient transfusion requirements, and guided by laboratory values (coagulation studies).

An interval assessment of hematoma volume (primary outcome measure) was accomplished by repeating a CT of the brain twenty-four hours from the patient's initial presentation. The volume of intracranial hemorrhage was again calculated using the $\mathrm{A} * \mathrm{~B} * \mathrm{C} / 2$ method by a blinded radiologist or un-blinded stroke team physician.

A trained research assistant recorded the secondary outcome measures of death and disability by accessing the patient's electronic medical record. The Modified Rankin Score was performed at the time of the patient's presentation to the ED and subsequently repeated by the stroke neurologist prior to discharge. This data was also obtained from the inpatient electronic medical record.

Cohort patient data was collected from our hospital stroke registry. A convenience sample of those patients with the diagnosis of ICH was then abstracted for comparison to the prospectively enrolled study population. A blinded stroke neurologist then calculated all of the hematoma volumes and Modified Rankin Scores for both groups.

\section{Results}

From June 2007 to June 2009 fifty patients were enrolled into the study. 19 patients were excluded for a failure to obtain a twenty-four hour head CT. The most common reason for failure to obtain the twenty-four hour head CT was emergent hematoma evacuation. Fifty patients were selected from a convenience sample from December 2009 to July 2010 to serve as a cohort group (Table 1). 33 patients were excluded from the control group because they needed emergent hematoma evacuation. The difference in mean total hematoma volume at twentyfour hours was $7.29 \mathrm{ml}$ (cohort) and $2.84 \mathrm{ml}$ (study). This resulted in an absolute decrease in hematoma size of $4.45 \mathrm{ml}$ in those patients who were treated aggressively with nicardapine within one hour of their presentation to the ED (Table 2). The time to reach our blood pressure target occurred more rapidly in the cohort group [150.9 to 91.4 min ( $>$ > 0.05)]. Sampling the ICH population following completion of a study is likely to suffer from retention of some of the study protocol as local standard practice for treating ICH patients has changed. We also found that the time from patient presentation in the ED to the initiation of a nicardapine infusion was significantly faster in the study group as compared to the cohort [51.83 to $84.7 \mathrm{~min}$ ( $\mathrm{p}>0.05$ )]. The Modified Rankin Scores were not significantly different between these two groups, but the study power was affected by the dismissal of patients from the cohort group who required emergent hematoma evacuation.

\section{Statistical Analysis Used}

A research assistant obtained all data from the patient's emergency department encounter and ICU stay by accessing the electronic medical record and using data collection sheets approved by the local IRB. The data was then transferred to an Excel $^{\mathrm{TM}}$ spreadsheet. The Microsoft ${ }^{\mathrm{TM}}$ spreadsheet was then analyzed using SPSS (Version 19) software. Then the data was processed using the TTEST procedure to allow for the Satterthwaite and Pooled methods to derive mean, standard deviation, standard error and to obtain the confidence intervals found 
Table 1. Frequency analysis of demographic data among participants of the ABC-ICH Trial $(\mathrm{N}=53$ cases, 36 comparison group).

\begin{tabular}{|c|c|c|c|c|c|c|c|c|c|}
\hline \multirow{2}{*}{$\begin{array}{c}\text { Variable } \\
\text { Age }\end{array}$} & \multirow[t]{2}{*}{ Category } & \multicolumn{2}{|c|}{$\mathbf{N}$} & \multicolumn{2}{|c|}{ Frequency \% } & \multicolumn{2}{|c|}{ Mean } & \multicolumn{2}{|c|}{ Missing } \\
\hline & & $\begin{array}{l}\text { Study } \\
\text { Group }\end{array}$ & $\begin{array}{l}\text { Cohort } \\
\text { Group }\end{array}$ & $\begin{array}{l}\text { Study } \\
\text { Group }\end{array}$ & $\begin{array}{l}\text { Cohort } \\
\text { Group }\end{array}$ & $\begin{array}{l}\text { Study } \\
\text { Group }\end{array}$ & $\begin{array}{l}\text { Cohort } \\
\text { Group }\end{array}$ & $\begin{array}{l}\text { Study } \\
\text { Group }\end{array}$ & $\begin{array}{l}\text { Cohort } \\
\text { Group }\end{array}$ \\
\hline & & & & & & 60.4 & 62.1 & 0 & 0 \\
\hline & $<55$ years & 19 & 12 & 35.8 & 33.3 & & & & \\
\hline & 56 - 65 years & 18 & 12 & 34.0 & 33.3 & & & & \\
\hline & 66 - 75 years & 7 & 7 & 13.2 & 19.4 & & & & \\
\hline & 76 - 85 years & 7 & 1 & 13.2 & 2.8 & & & & \\
\hline & $\geq 86$ years & 2 & 4 & 3.8 & 11.1 & & & & \\
\hline \multirow[t]{3}{*}{ Gender } & & & & & & -- & -- & 0 & 0 \\
\hline & Male & 27 & 20 & 50.9 & 55.6 & & & & \\
\hline & Female & 26 & 16 & 49.1 & 44.4 & & & & \\
\hline \multirow[t]{6}{*}{ Initial Systolic BP } & & & & & & 206.5 & 185.9 & 0 & 0 \\
\hline & $\begin{array}{l}\text { Stage } 1 \text { Hypertension } \\
\quad(\mathrm{SBP} \leq 140)\end{array}$ & 4 & 2 & 7.5 & 5.6 & & & & \\
\hline & $\begin{array}{l}\text { Stage } 2 \text { Hypertension } \\
\quad \text { (SBP } 141 \text { - 160) }\end{array}$ & 4 & 8 & 7.5 & 22.2 & & & & \\
\hline & $\begin{array}{l}\text { Stage } 3 \text { Hypertension } \\
\quad \text { (SBP } 161 \text { - 180) }\end{array}$ & 4 & 3 & 7.5 & 8.3 & & & & \\
\hline & $\begin{array}{l}\text { Stage } 4 \text { Hypertension } \\
\quad \text { (SBP } 181 \text { - 210) }\end{array}$ & 13 & 14 & 24.5 & 38.9 & & & & \\
\hline & $\begin{array}{c}\text { Exceeds Stage } 4 \\
\text { Hypertension }\end{array}$ & 28 & 9 & 52.8 & 25.0 & & & & \\
\hline \multirow[t]{6}{*}{ Initial Diastolic BP } & & & & & & 120.4 & 96.0 & 0 & 0 \\
\hline & $\begin{array}{l}\text { Stage } 1 \text { Hypertension } \\
(\mathrm{DBP} \leq 90)\end{array}$ & 4 & 18 & 7.5 & 50.0 & & & & \\
\hline & $\begin{array}{l}\text { Stage } 2 \text { Hypertension } \\
\quad \text { (SBP } 91 \text { - 100) }\end{array}$ & 9 & 4 & 17 & 11.1 & & & & \\
\hline & $\begin{array}{l}\text { Stage } 3 \text { Hypertension } \\
\quad \text { (SBP } 101 \text { - 110) }\end{array}$ & 9 & 5 & 17 & 13.9 & & & & \\
\hline & $\begin{array}{l}\text { Stage } 4 \text { Hypertension } \\
\text { (SBP } 111 \text { - 120) }\end{array}$ & 6 & 4 & 11.3 & 11.1 & & & & \\
\hline & $\begin{array}{l}\text { Exceeds Stage } 4 \\
\text { Hypertension }\end{array}$ & 25 & 5 & 47.2 & 13.9 & & & & \\
\hline \multirow[t]{3}{*}{ Controlled BP } & & & & & & -- & -- & 0 & 7 \\
\hline & Yes & 52 & 28 & 98.1 & 77.8 & & & & \\
\hline & No & 1 & 1 & 1.9 & 2.8 & & & & \\
\hline \multirow[t]{4}{*}{$\begin{array}{c}\text { Time to first dose of } \\
\text { Cardene }\end{array}$} & & & & & & 51.8 & 84.7 & 1 & 0 \\
\hline & No Cardene given & 0 & 9 & 0 & 25.0 & & & & \\
\hline & 1 - 60 minutes & 36 & 8 & 67.9 & 22.2 & & & & \\
\hline & 61 - 360 minutes & 16 & 18 & 30.2 & 50.0 & & & & \\
\hline \multirow[t]{4}{*}{ Time to control BP } & & & & & & 175.4 & 91.4 & 0 & 1 \\
\hline & BP control not reached & 0 & 1 & 0 & 2.8 & & & & \\
\hline & 1 - 60 minutes & 7 & 8 & 13.2 & 22.2 & & & & \\
\hline & 61 - 360 minutes & 42 & 18 & 79.2 & 50.0 & & & & \\
\hline
\end{tabular}




\begin{tabular}{|c|c|c|c|c|c|c|c|c|c|}
\hline & 361 - 600 minutes & 2 & 0 & 3.8 & 0 & & & & \\
\hline & 601 - 750 minutes & 1 & 0 & 1.9 & 0 & & & & \\
\hline \multirow[t]{8}{*}{ Modified Rankin Score } & & & & & & 4.2 & 3.7 & 10 & 2 \\
\hline & 0 & 1 & 1 & 1.9 & 2.8 & & & & \\
\hline & 1 & 2 & 1 & 3.8 & 2.8 & & & & \\
\hline & 2 & 3 & 7 & 5.7 & 19.4 & & & & \\
\hline & 3 & 4 & 5 & 7.5 & 13.9 & & & & \\
\hline & 4 & 13 & 10 & 24.5 & 27.8 & & & & \\
\hline & 5 & 12 & 3 & 22.6 & 8.3 & & & & \\
\hline & 6 & 8 & 7 & 15.1 & 19.4 & & & & \\
\hline
\end{tabular}

in Table 2.

Our primary outcome was: a measurable decrease in hematoma volume seen on the twenty-four hour brain CT in those patients who received an early infusion of nicardapine (within one hour of their presentation to the ED) for aggressive blood pressure reduction. Secondary outcomes included an improved Modified Rankin Score, NIHSS and decrease in mortality.

\section{Limitations}

The primary limitation of this study was our ability to enroll a sufficient number of patients to power our results for clinical outcomes. Given the severity of this disease process, the majority of the cohort patients were placed into one of two categories that often precluded their continued enrollment in this study: emergent hematoma evacuation and impending death. This study would have to be repeated over a longer enrollment period or in concordance with a multi-center approach to show meaningful clinical outcomes in secondary measures such as the Modified Rankin Scores. A repeated study with a more robust enrollment would also be needed to evaluate the trend seen in this study towards decreased hematoma size post ICH with the early initiation of nicardipine infusions. It would also be extremely useful to try and ascertain if the initiation of some form of blood pressure reduction agent is more important than the total time it takes to achieve blood pressure reduction. Another constraint that affected our outcomes was that the in-house stroke team and Houston Emergency Medicine Service had preexisting protocols for rapidly lowering systolic blood pressures to a target of $<180$ with labetolol for all suspected stroke patients. Their protocols were designed to assure that the more commonly encountered patients with ischemic stroke also meet criteria for fibrinolysis if there was no CT evidence of intracerebral hemorrhage. Eight patients in the cohort group also received blood pressure therapy in their initial hour of presentation to the ED (Table 1). Per our hypothesis, we would then expect those patients in the cohort group who received early blood pressure therapy to have had a decreased twenty-four hour hematoma volume and improved Modified Rankin Score, which they did. Ideally, the study would need to be repeated utilizing only one blood pressure optimizing agent.

\section{Discussion}

Intracerebral hemorrhage is a known complication of longstanding hypertension and usually results in rupture of a small vessel in the cerebellum, thalamus, pons, or basal ganglion. Modern neuro-imaging studies indicate that continued bleeding and expansion of the hematoma occurs in up to one third of patients within the first several hours of onset, and probably over three to twenty-four hours in another ten percent [3] [4]. Further neurological deterioration may occur over several days secondary to the adverse effects of edema and inflammation in the peri-hematomal region [5]. Most cases of ICH are attributed to chronic hypertension and the vast majority of these patients are severely hypertensive in the first twenty-four hours of their stroke. The volume of the hematoma is a critical determinant of mortality and functional outcome after intracerebral hemorrhage, and early hematoma growth is an important cause of neurologic deterioration [3] [11] [16]. An increase in volume of more than thirty-three percent is detectable on repeated computed tomography (CT) in thirty-eight percent of patients initially 
Table 2. Comparison of Means of Hematoma Size between Cases and Comparison Group by using an Independent Group Ttests.

\begin{tabular}{|c|c|c|c|c|c|c|}
\hline $\mathbf{N}$ & Mean & Standard Deviation & Standard Error & Minimum & Maximum & \\
\hline 17 & 7.29 & 20.62 & 5.00 & -28.3 & 56.30 & \\
\hline \multirow[t]{2}{*}{31} & 2.85 & 14.67 & 2.63 & -19.20 & 74.90 & \\
\hline & 4.45 & 16.97 & 5.12 & & & \\
\hline \multirow[t]{3}{*}{ Method } & Mean & $95 \%$ CI & Mean & $\begin{array}{l}\text { Standard } \\
\text { Deviation }\end{array}$ & $95 \%$ CI & $\begin{array}{l}\text { Standard } \\
\text { Deviation }\end{array}$ \\
\hline & 7.29 & -3.31 & 17.89 & 20.62 & 15.36 & 31.38 \\
\hline & 2.85 & -2.53 & 8.23 & 14.67 & 11.72 & 19.60 \\
\hline Pooled & 4.45 & -5.87 & 14.76 & 16.97 & 14.11 & 21.32 \\
\hline Satterthwaite & 4.45 & -7.19 & 16.08 & & & \\
\hline Method & Variances & $\mathrm{DF}$ & $\mathrm{T}$ Value & $\operatorname{Pr}>|t|$ & & \\
\hline Pooled & Equal & 46 & 0.87 & 0.39 & & \\
\hline \multirow[t]{2}{*}{ Satterthwaite } & Unequal & 25.08 & 0.79 & 0.45 & & \\
\hline & \multicolumn{4}{|c|}{ Equality of Variances } & & \\
\hline Folded F & 16 & 30 & 1.98 & 0.10 & & \\
\hline
\end{tabular}

scanned within the first three hours of onset of their symptoms; in two thirds of these cases this change is noticeable on CT within the first hour [10] [11]. This suggests that early aggressive blood pressure reduction would decrease hematoma size and edema. This is further supported by the fact that patients with high blood pressure and acute intracerebral hemorrhage have worse outcomes than their counterparts [1].

Early hematoma growth occurs in the absence of a measurable coagulopathy and appears to result from continued bleeding or re-bleeding at multiple sites within the first few hours after onset. Early hematoma growth may be exacerbated by arterial hypertension and by local coagulopathy induced by the hematoma [1]-[5].

Since large multicenter trials designed to limit the hematoma size in ICH by increasing coagulability have failed [17], blood pressure control appears to be the remaining opportunity to affect this condition in its acute phase. The Factor Seven for Acue Hemorrhagic Stroke Trial (FAST) has cast some doubt on the importance of hematoma control. FAST detected no improvement in survival or functional outcomes with small reported hematoma growth (average 2.6 to $3.8 \mathrm{ml}$ over twenty-four hours) when participants were treated with recombinant activated Factor VII [17]. Although there was no overall mortality benefit, subgroup analysis has shown some modest benefit in certain populations to include those treated in less than 2.5 hours [18]. It has recently been proposed that confounders like prognostic factors between randomized groups, comorbid effects of residual disability in older patients, and adverse thromboembolic events contributed to a type one error [18]. Other observational studies however, had previously established that early reduction in blood pressure was associated with smaller hematoma volumes [3] [11] [16].

There were two clinical trials (INTERACT and ATACH) published in 2010, which were designed to identify a target blood pressure that would limit the expansion of hematoma volume [19] [20]. However, hematoma size and growth are disease oriented outcomes and the question of patient oriented benefit is still largely unanswered.

The Antihypertensive Treatment of Acute Cerebral Hemorrhage (ATACH) study was a randomized control trial designed to assess the safety of lowering blood pressures in ICH patients into one of three tiers (tier one SBP $\leq 200$ and $\geq 170$, tier two SBP $<170$ and $\geq 140$, and tier three SBP $<140$ and $\geq 110$ ) by using nicardipine. [16] The mean time to initiate nicardapine was approximately three hours. The patients in the second and third tiers had small hematomas, but they also had a trend toward a higher incidence of serious adverse events although those events did not reach clinical significance [19]. This study was not powered to uncover clinical outcome measures, but again there was no significant change in the Modified Rankin Score between the different patient groups, but the study was not powered to assess clinical or statistical outcome measures [19].

The Intensive Blood Pressure Reduction in Acute Cerebral Haemorrhage Trial (INTERACT) was a randomized controlled trial that proved early initial blood pressure control was safe and well tolerated [20]. 
INTERACT randomized 404 patients with ICH and elevated SBP $(<220$ and $\geq 150)$ to either intensive blood pressure reduction (SBP $<140$ ) or to the American Stroke Association guideline (SBP $<180$ ) within six hours of presentation to the ED. The primary endpoint was change in hematoma volume at twenty-four hours [20]. The study was not powered to differentiate between clinical outcomes, but follow up at three months showed no significant difference in mortality between the two groups. However, in subgroup analysis hematoma expansion was significantly decreased by aggressive blood pressure control in patients recruited within three hours and in those patients with an initial SBP $\geq 181$ [20]. The majority of the time, this was accomplished using intravenous loop diuretics or ace inhibitors to lower systolic blood pressure, but occasionally oral medications were also employed.

There were numerous key differences between these studies, to include an overall lower enrollment SBP in the INTERACT study (only $47 \%$ with SBP $\geq 180$ ), ethnicity, choice of blood pressure medications and medication delivery [19] [20]. INTERACT chose to include $10 \%$ of those with a CT diagnosis of non-supratentoral bleeds in their post hoc analysis, which would also be less likely to be of hypertensive origin [19]. ATACH had a much higher success rate at early blood pressure control (90\% of those recruited were controlled at 2 hours). This could be attributed to their study medication choice [20]. These differences may explain the variation in the two studies, but they do not explain the failure of either study to reach a clinical significance by limiting the size of hematoma volume. It is likely that a large multicenter trial will be necessary to ascertain the clinical significance of limiting hematoma volume with aggressive early blood pressure reduction because even the highest volume emergency departments in the United States would need several years to collect enough patients with intracerebral hemorrhage to generate the power to find these differences.

Both INTERACT and ATACH have provided the necessary background to support the necessity of the ABC ICH trial. Both call for a prospective study to evaluate reduction in hematoma volume and outcome benefits in patients who have their blood pressure aggressively controlled in less than four hours from onset of symptoms.

There is conflicting evidence from prospective studies, retrospective studies and case series regarding the efficacy of blood pressure reduction in decreasing the rate of early hematoma growth. We hypothesized that initiation of prompt and aggressive blood pressure reduction in acute ICH patients will result in a reduction of early hematoma growth.

The primary outcome of this study was: a measurable decrease in hematoma volume in those patients who received an early infusion of nicardapine (within one hour of their presentation to the ED) for aggressive blood pressure reduction. Our data points toward a trend in smaller hematoma volumes (absolute decrease $4.45 \mathrm{ml}$ ) in those patients receiving early aggressive blood pressure therapy, but due to a limited sample size and variable hematoma sizes the confidence intervals overlap zero. A larger sample size would be required to establish that a reliable reduction in ICH volume occurs for those patients who not only receive an early nicardapine infusion (sixty minutes from presentation), but are then able to maintain those optimized blood pressure parameters. Further, the same sample size would be needed to delineate what that ideal blood pressure is and how quickly it should be achieved to maximize clinical outcomes. These results support the previous research hypothesis (INTERACT and ATACH) that aggressive blood pressure control in intracerebral hemorrhage may show some statistical benefit in the reduction of hematoma size, however a larger sample size would be necessary to show clinical significance.

\section{Conclusion}

The results of the ABC-ICH trial support previous studies (the INTERACT and ATACH trials) suggesting that early aggressive blood pressure control in intracerebral hemorrhage reduces hematoma growth, however the clinical benefit of such reduction will have to be evaluated in future trials. The ABC-ICH trial reinforces the current thinking that the majority of patients with intracerebral hemorrhage, which was not so large as to warrant an immediate hematoma evacuation, have a disease that may benefit from early aggressive blood pressure reduction with respect to limiting the size of hematoma expansion.

\section{References}

[1] Qureshi, A.I., Tuhrim, S., Broderick, J.P., Batjer, H.H., Hondo, H. and Hanley, D.F. (2001) Spontaneous Intracerebral Hemorrhage. The New England Journal of Medicine, 344, 1450-1460. http://dx.doi.org/10.1056/NEJM200105103441907 
[2] Flaherty, M.L., Haverbusch, M., Sekar, P., Kissela, B., Kleindorfer, D., Moomaw, C.J., Sauerbeck, L., et al. (2006) Long-Term Mortality after Intracerebral Hemorrhage. Neurology, 66, 1182-1186. http://dx.doi.org/10.1212/01.wnl.0000208400.08722.7c

[3] Broderick, J.P., Brott, T.G., Tomsick, T., et al. (1990) Ultra-Early Evaluation of Intracerebral Hemorrhage. Journal of Neurosurgery, 72, 195-199. http://dx.doi.org/10.3171/jns.1990.72.2.0195

[4] Chen, S.T., Chen, S.D., Hsu, C.Y., et al. (1989) Progression of Hypertensive Intracerebral Hemorrhage. Neurology, 39, 1509-1514. http://dx.doi.org/10.1212/WNL.39.11.1509

[5] Vemmos, K.N., Tsivgoulis, G., Spengos, K., et al. (2003) Association between 24-h Blood Pressure Monitoring Variables and Brain Edema in Patients with Hyperacute Stroke. Journal of Hypertension, 21, 2167-2173. http://dx.doi.org/10.1097/00004872-200311000-00027

[6] Dandapani, B.K., Suzuki, S., Kelley, R.E., et al. (1995) Relation between Blood Pressure and Outcome in Intracerebral Hemorrhage. Stroke, 26, 21-24. http://dx.doi.org/10.1161/01.STR.26.1.21

[7] Broderick, J.P., Diringer, M.N., Hill, M.D., et al. (2007) Determinants of Intracerebral Hemorrhage Growth: An Exploratory Analysis. Stroke, 38, 1072-1075. http://dx.doi.org/10.1161/01.STR.0000258078.35316.30

[8] Jauch, E.C., Lindsell, C.J., Adeoye, O., et al. (2006) Lack of Evidence for an Association between Hemodynamic Variables and Hematoma Growth in Spontaneous Intracerebral Hemorrhage. Stroke, 37, 2061-2065. http://dx.doi.org/10.1161/01.STR.0000229878.93759.a2

[9] Qureshi, A.I., Tuhrim, S., Broderick, J.P., et al. (2001) Spontaneous Intracerebral Hemorrhage. The New England Journal of Medicine, 344, 1450-1460. http://dx.doi.org/10.1056/NEJM200105103441907

[10] Broderick, J.P., Brott, T.G., Tomsick, T., Barsan, W. and Spilker, J. (1990) Ultra-Early Evaluation of Intracerebral Hemorrhage. Journal of Neurosurgery, 72, 195-199. http://dx.doi.org/10.3171/jns.1990.72.2.0195

[11] Kazui, S., Minematsu, K., Yamamoto, H., Sawada, T. and Yamaguchi, T. (1997) Predisposing Factors to Enlargement of Spontaneous Intracerebral Hematoma. Stroke, 28, 2370-2375. http://dx.doi.org/10.1161/01.STR.28.12.2370

[12] Kim-Han, J.S., Kopp, S.J., Dugan, L.L., et al. (2006) Perihematomal Mitochondrial Dysfunction after Intracerebral Hemorrhage. Stroke, 37, 2457-2462. http://dx.doi.org/10.1161/01.STR.0000240674.99945.4e

[13] Powers, W.J., Zazulia, A.R., Videen, T.O., et al. (2001) Autoregulation of Cerebral Blood Flow Surrounding Acute (6 to 22 Hours) Intracerebral Hemorrhage. Neurology, 57, 18-24. http://dx.doi.org/10.1212/WNL.57.1.18

[14] Broderick, J., Connolly, S., Feldmann, E., et al. Guidelines for the Management of Spontaneous Intracerebral Hemorrhage in Adults: 2007 Update: A Guideline from the American Heart Association/American Stroke Association Stroke Council, High Blood Pressure Research Council, and the Quality of Care and Outcomes in Research Interdisciplinary Working Group Stroke. J Cerebral Circ, 2007, 38, 2001-2023; Critical Care Medicine, 38, 2010, 643.

[15] Steiner, T., Kaste, M., Forsting, M., et al. (2006) Recommendations for the Management of Intracranial Haemorrhage-Part I: Spontaneous Intracerebral Haemorrhage. The European Stroke Initiative Writing Committee and the Writing Committee for the EUSI Executive Committee. Cerebrovascular Disease, 22, 294-316. http://dx.doi.org/10.1159/000094831

[16] Ohwaki, K., Yono, E., Nagashima, H., Hirata, M., Nakagomi, T. and Tamura, A. (2004) Blood Pressure Management in Acute Intracerebral Hemorrhage: Relationship between Elevated Blood Pressure and Hematoma Enlargement. Stroke, 35, 1364-1367. http://dx.doi.org/10.1161/01.STR.0000128795.38283.4b

[17] Mayer, S.A., Brun, N.C., Begtrup, K., Broderick, J., Davis, S., Diringer, M.N., et al. (2008) Efficacy and Safety of Recombinant Activated Factor VII for Acute Intracerebral Hemorrhage. The New England Journal of Medicine, 358, 2127-2137. http://dx.doi.org/10.1056/NEJMoa0707534

[18] Mayer, S.A., Davis, S.M., Skolnick, B.E., Brun, N.C., Begtrup, K., Broderick, J.P., et al. (2009) Can a Subset of Intracerebral Hemorrhage Patients Benefit from Hemostatic Therapy with Recombinant Activated Factor VII? Stroke, 40, 833-840. http://dx.doi.org/10.1161/STROKEAHA.108.524470

[19] Qureshi, A.I. (2010) Antihypertensive Treatment of Acute Cerebral Hemorrhage (ATACH). Critical Care Medicine, 38, 637-648. http://dx.doi.org/10.1097/CCM.0b013e3181b9e1a5

[20] Anderson, C.S., Huang, Y., Wang, J.G., et al. (2010) INTERACT Investigators. Intensive Blood Pressure Reduction in Acute Cerebral Hemorrhage Trial (INTERACT). Stroke, 41, 307-312. http://dx.doi.org/10.1161/STROKEAHA.109.561795 